\title{
Qualidade de Vida em Portadores de Gonartrose Submetidos a Liberação Miofascial
}

\author{
Laís Oliveira Pithon \\ Acadêmica do $8^{\circ}$ semestre do curso de Fisioterapia \\ da Escola Bahiana de Medicina e Saúde Pública \\ (laipithon@gmail.com) \\ Silvana Almeida Nascimento Ribas \\ Fisioterapeuta e especialista em clínica de dor \\ Tipo de Pesquisa \\ Origem da Pesquisa \\ Artigo Original \\ Trabalho de Conclusão de Curso de Fisioterapia da \\ Análise do Manuscrito \\ Escola Bahiana de Medicina e Saúde Pública. ${ }^{1}$ \\ Corpo Editorial da Bahiana \\ Tipo de Análise do Manuscrito \\ Peer Blind Review \\ Recebido em Mai/11 \\ Aprovado em Jun/11
}

\section{Resumo}

Introdução: A liberação miofascial (LM) é uma técnica utilizada com o objetivo de melhorar a função corporal, reorganização postural, reduzir dor, além de proporcionar relaxamento emocional e físico. Apesar da existência de estudos que verificam impacto positivo em diversas patologias, esses ainda são inconclusivos para evidência de eficácia da LM como tratamento para Gonartrose. A gonartrose, por sua vez, torna-se mais freqüente no mundo, associada à idade avançada e estilo de vida. Por ser uma patologia crônica e degenerativa, mostra-se a importância da identificação de tratamentos que possam ter impacto positivo na qualidade de vida (QV) dos mesmos. Objetivo: Este estudo tem o objetivo de identificar impacto da LM na QV dos pacientes que possuem gonartrose em uma clínica de fisioterapia em Salvador-Bahia. Métodos: O estudo foi um ensaio clínico, longitudinal e paramétrico. Foi aplicado protocolo de LM adaptado pelo autor no período de 9 sessões, com a freqüência de duas vezes por semana. Para verificação do impacto na $Q V$, foi identificado o escore do questionário SF-36 antes e após a intervenção. Para análise estatística foram utilizados os testes Wilcoxon e Qui-Quadrado. Resultados: A amostra foi representada por 13 indivíduos, em que $11(84,6 \%)$ eram do sexo feminino, cuja mediana de idade foi de 67 anos $(58,00$ 73,00 ) e predominaram indivíduos com obesidade classe I e III. Houve significância estatística nos domínios capacidade funcional $(\mathrm{P}=0,042)$, dor $(\mathrm{P}=0,003)$ e aspectos sociais $(\mathrm{P}=0,019)$ do questionário SF-36. A mediana de melhora nestes aspectos foi de $20(0,00-$ $65,00), 14(0,00-79,00)$ e $25(0,00-50,00)$ pontos respectivamente. Conclusão: A LM demonstrou impacto na QV na amostra, representando significância estatística na melhora da capacidade funcional, dor e aspectos sociais. Mais estudos devem ser reproduzidos para determinar em outras populações se a técnica também possui impacto na qualidade de vida.

Palavras-chave: Osteoartrite de joelho, fascia, tecido conjuntivo, qualidade de vida

${ }^{1}$ Este trabalho foi aprovado pelo Comitê de Ética e Pesquisa da Escola Bahiana de Medicina e Saúde Pública, cujo protocolo é n⿳0138/2010 


\title{
Quality of Life in Patients Undergoing gonarthrosis Myofascial Release
}

\begin{abstract}
Introduction: The Myofascial Release (LM) is a technique used for the purpose of improving bodily function, postural reorganization, reduce pain, and provide emotional and physical relaxation. Although there are studies that verify positive impact in several diseases, they are still inconclusive evidence for the effectiveness of SCI as a treatment for gonarthrosis. The gonarthrosis, in turn, becomes more frequent in the world, associated with old age and lifestyle. Because it is a chronic degenerative disease, shows the importance of identifying treatments that may impact positively on the quality of life (QOL) of them. Objective: This study aims to identify the impact of LM on the QOL of patients who have gonarthrosis in a physical therapy clinic in Salvador, Bahia. Methods: The study was a clinical trial, longitudinal and parametric. LM was applied protocol adapted by the author in the period of 9 sessions as often as twice a week. To check the impact on QoL, was identified score of the SF-36 before and after the intervention. The statistical analysis used the Wilcoxon and chi-square. Results: The sample was represented by 13 individuals, in which $11(84.6 \%)$ were females, whose median age was 67 years (from 58.00 to 73.00 ) and obese subjects were predominant class I and III . There was statistical significance in the field capacity $(\mathrm{P}=0.042)$, pain $(\mathrm{P}=0.003)$ and social $(P=0.019)$ of the SF-36. The median improvement in these aspects was $20(0.00$ to $65.00), 14(0.00$ to 79.00$)$ and $25(0.00$ to 50.00$)$ points, respectively. Conclusion: QV LM demonstrated an impact on the sample, representing a statistically significant improvement in functional capacity, pain and social aspects. Further studies should be replicated in other populations to determine if the technique also has impact on quality of life.
\end{abstract}

Keywords: Osteoarthritis of the knee, fascia, connective tissue, quality of life

\section{Calidad de vida en pacientes intervenidos de lanzamiento gonartrosis miofascial}

\section{Resumen}

Introducción: El alivio miofascial (LM) es una técnica utilizada con el propósito de mejorar la función corporal, la reorganización de la postura, reducir el dolor y facilitar la relajación física y emocional. Aunque hay estudios que confirman el impacto positivo en varias enfermedades, son todavía pruebas concluyentes de la eficacia de la lesión medular como tratamiento de la gonartrosis. La gonartrosis, a su vez, se hace más frecuente en el mundo, asociados con la edad y estilo de vida. Debido a que es una enfermedad degenerativa crónica, muestra la importancia de identificar los tratamientos que pueden influir positivamente en la calidad de vida (QOL) de ellos. Objetivo: Este estudio tiene como objetivo identificar el impacto de la LM en la calidad de vida de los pacientes con gonartrosis en una clínica de fisioterapia en Salvador, Bahía. Métodos: El estudio fue un ensayo clínico, longitudinal y paramétricos. LM se aplicó el protocolo adaptado por el autor en el período de nueve sesiones de hasta dos veces a la semana. Para comprobar el impacto en la calidad de vida, fue identificado puntuación del SF-36 antes y después de la intervención. El análisis estadístico utilizó el test de Wilcoxon y el chi-cuadrado. Resultados: La muestra estuvo representada por 13 personas, de las cuales 11 $(84,6 \%)$ eran mujeres, cuya edad media fue de 67 años $(58,00$ a 73,00$)$ y los sujetos obesos eran de clase predominante I y III . No hubo significación estadística en la capacidad de campo $(P=0,042)$, dolor $(p=0,003)$ y social $(p=0,019)$ de la $S F-36$. La mediana de mejoría en estos aspectos fue de $20(0,00$ a 65,00$), 14(0.00-79.00)$ y $25(0,00$ a 50,00$)$ puntos, respectivamente. Conclusión: QV LM demostrado un impacto en la muestra, lo que representa una mejoría estadísticamente significativa en la capacidad funcional, el dolor y los aspectos sociales. Los estudios futuros deben ser replicados en otras poblaciones para determinar si la técnica también tiene impacto en la calidad de vida.

Palabras clave: La osteoartritis de la rodilla, la fascia, el tejido conectivo, la calidad de vida 


\section{Qualité de vie chez les patients subissant Communiqué de gonarthrose myofasciale}

\section{Résumé}

Introduction: Le Myofascial Release (LM) est une technique utilisée dans le but d'améliorer les fonctions corporelles, la réorganisation posturale, réduire la douleur, et fournissent une détente physique et émotionnelle. Bien qu'il existe des études qui permettent de vérifier l'impact positif dans plusieurs maladies, ils sont encore des preuves concluantes de l'efficacité de la SCI en tant que traitement pour la gonarthrose. La gonarthrose, à son tour, devient plus fréquente dans le monde, associée à la vieillesse et la vie. Parce qu'elle est une maladie dégénérative chronique, montre l'importance d'identifier les traitements qui peuvent influer positivement sur la qualité de vie (QV) d'entre eux. Objectif: Cette étude vise à identifier l'impact des LM sur la QV des patients qui ont une gonarthrose dans une clinique de physiothérapie à Salvador de Bahia. Méthodes: L'étude était un essai clinique, longitudinale et paramétrique. LM a été appliqué le protocole adapté par l'auteur dans la période de neuf séances aussi souvent que deux fois par semaine. Pour vérifier l'impact sur la qualité de vie, a été identifié score du SF-36 avant et après l'intervention. L'analyse statistique a utilisé le Wilcoxon et du chi-carré. Résultats: L'échantillon a été représenté par 13 personnes, dont 11 $(84,6 \%)$ étaient des femelles, dont l'âge médian était de 67 ans (de 58,00 à 73,00) et les sujets obèses ont la classe dominante I et III . Il y avait une signification statistique dans la capacité au champ $(P=0,042)$, de la douleur $(p=0,003)$ et sociales $(P=0,019)$ du SF-36. L'amélioration médiane dans ces aspects a été de $20(0.00$ à 65.00), $14(0.00$ à 79.00$)$ et 25 $(0,00$ à 50,00$)$ points, respectivement. Conclusion: QV LM a démontré un impact sur l'échantillon, ce qui représente une amélioration statistiquement significative de la capacité fonctionnelle, la douleur et les aspects sociaux. Des études complémentaires devraient être reproduits dans d'autres populations afin de déterminer si la technique a aussi un impact sur la qualité de vie.

Mots-clés: Arthrose du genou, le fascia, le tissu conjonctif, la qualité de vie

\section{Introdução}

A liberação miofascial (LM), ou massagem miofascial, tem sido cada vez mais utilizada pelos fisioterapeutas, assim como seus efeitos têm sido melhor explorados pela literatura. ${ }^{1}$

Considerada como terapêutica manual, esta técnica tem como objetivo liberar zonas de aprisionamento fasciais do corpo, facilitando seu alongamento, diminuindo a tensão no tecido e favorecendo assim, maior funcionalidade. ${ }^{1-4}$ Proporciona ainda o transporte de nutrientes para a fáscia, além da manutenção da homeostase tecidual.1,2

As patologias músculo-esqueléticas têm sido cada vez mais prevalentes, sendo relacionadas à idade avançada e estilo de vida. ${ }^{5}$ Seu impacto negativo na funcionalidade pode afetar estado psicossocial e econômico do indivíduo, assim como de sua família e cuidadores. ${ }^{6}$

A gonartrose está entre estas patologias e é caracterizada por uma degeneração progressiva da cartilagem articular do joelho.5,6 Seu quadro clínico envolve dor, edema, rigidez, crepitações, redução de amplitude articular, perda de funcionalidade e menor qualidade de vida.1,5,7,8 Sua prevalência, segundo à Organização Mundial de Saúde (OMS) é de aproximadamente 30 mil casos para 100 mil habitantes do sexo feminino e cerca de 23 mil casos para 100 mil habitantes do sexo masculino, sendo considerados esses dados para países em desenvolvimento da América. ${ }^{6}$

0 estímulo tensional (constante e prejudicial) miofascial é comum em patologias crônicas 3 , como a gonartrose, devido a sua patogênese. Esta tensão pode ser agravada com a restrição de movimentos ${ }^{9}$ inerente do sedentarismo (em que os indivíduos que possuem gonartrose possuem tendência de se tornarem). ${ }^{10}$ 
Em patologias crônicas (onde a cura nem sempre é possível e há necessidade de gestão de sua condição) a qualidade de vida torna-se um importante indicador de saúde, abrangendo o indivíduo em seu contexto sócio-político-cultural.11,12

Estudos mostram que a LM possui impacto na qualidade de vida.13,14 Dentre outros benefícios relatados na literatura, há melhora da dor e função física.14,15 Alguns autores ainda sugerem que a melhora da função gera melhor qualidade de vida. ${ }^{13}$ Apesar de não haver uma evidência conclusiva da efetividade da LM para tratamento da gonartrose, sua evidência é favorável, levantando a necessidade de mais pesquisas. ${ }^{16}$

Considerando que a tendência dos próximos anos é que se obtenha uma população majoritariamente de idosos, devido ao aumento da expectativa de vida, possivelmente essa doença será mais prevalente. 6 Diante disso, o presente estudo tem o objetivo de comparar a qualidade de vida em pacientes que possuem gonartrose, antes e após serem submetidos a um protocolo de liberação miofascial.

\section{Material \& Método}

0 estudo foi um ensaio clínico, cuja população acessível foi de pacientes de uma clínica de fisioterapia, portadores de gonartrose, ambos os sexos, acima de 18 anos. Os voluntários foram selecionados através da identificação do Código Internacional de Doenças (CID) correspondente à gonartrose (M17).

Os critérios de inclusão foram pacientes diagnosticados com Artrose em joelho, sem neoplasias e que não fossem gestantes. Os critérios de exclusão foram voluntários com dermatites, úlceras ou alodínia no local de intervenção e sem possibilidade de ir à clínica de fisioterapia. Não foram incluídos indivíduos que se negaram a assinar o Termo de Consentimento Livre e Esclarecido.

o cálculo amostral foi realizado na calculadora do Laboratório de Epidemiologia e Estatística (LEE), criada pela Faculdade de Medicina da Universidade de São Paulo (USP). ${ }^{17}$ A média de desvio padrão utilizada foi 21 , diferença relevante nos estudos de 20 e nível de significância de $5 \%$, sendo obtido o poder de estudo de $80 \%$ e o número de 14 voluntários. Com 12 voluntários o poder do estudo tornou-se de $75 \%$.

A variável de desfecho é a qualidade de vida dos voluntários. 0 preditor foi o protocolo de liberação miofascial. As variáveis de interesse foram idade, sexo e Índice de Massa Corpórea (IMC). ${ }^{18}$

Para avaliação da QV, foi utilizado o questionário genérico SF-36 (versão em português) antes e após o período de intervenção. Antes do protocolo foram também registrados idade, altura, peso e calculado IMC (com fórmula Peso/ Altura ${ }^{2}$ ). 0 instrumento utilizado foi a balança eletrônica com estadiômetro da marca Welmy, que possui capacidade máxima de 200 $\mathrm{kg}$.

\section{Protocolo de LM:}

Foram realizadas sessões com freqüência de duas vezes por semana, que aconteceram no período total de um mês ( 9 sessões), entre 01/02/11 e 01/03/11, com duração de 30 minutos cada. A técnica ocorreu entre $7: 00 \mathrm{~h}$ e 12:00h da manhã e foi realizada por três estudantes do último ano de fisioterapia, previamente treinadas.

Cada sessão diária incluiu duas técnicas de LM, abrangendo na primeira Isquiotibiais e Quadríceps e na segunda, englobando cadeira posterior e anterior dos membros inferiores, de forma mais global.

A primeira técnica consiste em um deslizamento no sentido das fibras musculares, utilizando as articulações interfalangeanas médias, da segunda à quinta falange do terapeuta. ${ }^{1,19}$ Foi realizada de forma lenta e gradual, com menor profundidade da pressão e 
menor velocidade do movimento nas áreas mais hipercontraídas, evitando hiperextensão de punhos (do terapeuta).

Em região posterior e lateral do membro inferior, o pesquisador posiciona-se ao lado ipsilateral à região a ser trabalhada, enquanto o voluntário posiciona-se em decúbito ventral, com braços ao longo do corpo. 0 deslizamento nesta posição se faz caudalmente19 (Figura 1A e 1-B).

Já em região anterior e medial do membro inferior, o pesquisador posiciona-se ao lado ipsilateral ao joelho a ser trabalhado, enquanto o voluntário posiciona-se em decúbito dorsal, com braços ao longo do corpo e com joelhos apoiados sob uma almofada. 0 pesquisador então realiza deslizamento no sentido cranial 19 (Figura 1-C e 1-D).

A segunda técnica consiste em um alongamento suave, em que as duas mãos envolvem e alongam a fáscia, movendo-a de acordo com suas inclinações próprias que vão sentidas pelas mãos. A fáscia possui seus próprios micromovimentos, estes que guiaram esta segunda técnica.19,20

0 terapeuta posiciona-se ipsilateralmente ao membro afetado, com mão cefálica no terço médio da tíbia e mão caudal na articulação tibiotársica. 0 voluntário posiciona-se em decúbito dorsal, com braços ao longo do corpo e almofada apoiada sob os joelhos. É então realizada uma tração do tecido fascial, com consequente tração articular, acompanhando os movimentos fasciais. ${ }^{19} \mathrm{Na}$ mesma sessão, com o ganho de amplitude, vai retirando-se a almofada para garantir alongamento das musculaturas (Figura 1-E).

Os terapeutas foram treinados anteriormente à aplicação do protocolo, para evitar viés metodológico.

Figura 1.

Protocolo de liberação miofascial.

A= Isquitibiais. $B=$ Abdutores. $C=$ Quadríceps. $D=$ Adutores. $E=$ Membro inferior

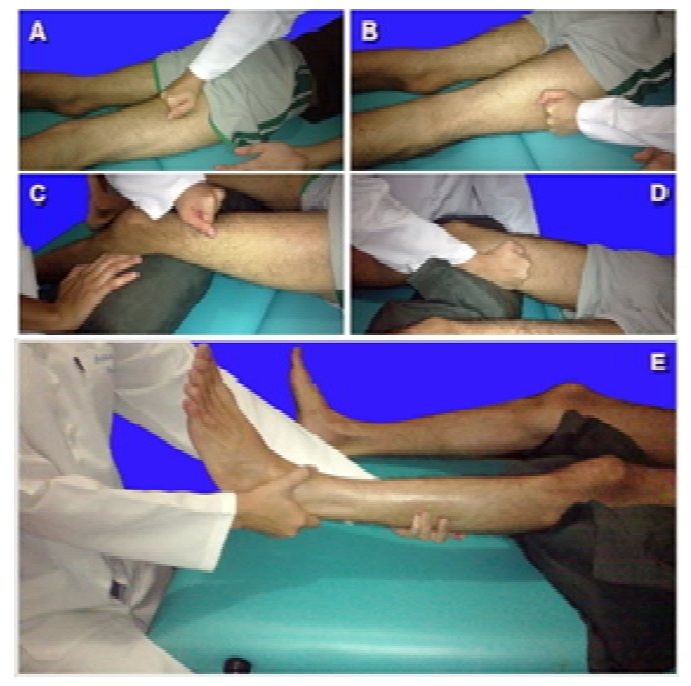

A análise de dados foi feita através do Statistical Package for The Social Sciences (SPSS) 14.0 for Windows, utilizando Teste Wilcoxon. 


\section{Resultados}

Foram recrutados 22 indivíduos, em que oito foram excluídos, pois se negaram a assinar o TCLE, permanecendo 14 voluntários no estudo. Destes, houve perda de segmento de um voluntário, por não concluir o período proposto de intervenção. Esta população apresentou distribuição assimétrica, portanto foram utilizadas medianas e intervalos interquartis para representá-las.

A população foi composta por 13 indivíduos, sendo $11(84,60 \%)$ do sexo feminino. A mediana das idades foi de idades 67 anos $(58,00-73,00)$, altura média de $1,50 \mathrm{~cm}( \pm 0,08)$ e peso com média de $71,62 \mathrm{Kg}( \pm 15,91)$.

Em relação ao IMC, apenas um indivíduo $(7,70 \%)$ apresentou peso eutrófico, cinco indivíduos $(38,50 \%)$ obtiveram sobrepeso e os demais, apresentavam obesidade classe I e classe III (53,90\%; sete indivíduos).

A análise estatística revelou impacto na QV após LM, com significância estatística nos domínios capacidade funcional $(\mathrm{P}=0,042)$, dor $(\mathrm{P}=0,003)$ e aspectos sociais $(\mathrm{P}=0,019)$ (Tabela 1).

Foi identificado aumento no escore do questionário SF-36 em: 11 voluntários $(84,60 \%)$ no domínio dor, 9 dos voluntários $(69,20 \%)$ no domínio capacidade funcional e em 10 dos voluntários $(76,90 \%)$ no domínio aspectos sociais.

Tabela 1

Domínios do SF-36, pré-intervenção e pós-intervenção, em população com gonartrose, Salvador-Bahia, 2011

\begin{tabular}{l|c|c|c}
\multicolumn{1}{c|}{ Domínios } & MPI & MPOI & P valor \\
\hline Capacidade Funcional & $25,00(20,00-32,50)$ & $50,00(35,00-65,00)$ & 0,042 \\
\hline Aspectos Físicos & $00,00(0,00-50,00)$ & $25,00(12,50-62,50)$ & 0,189 \\
\hline Dor & $41,00(11,00-51,00)$ & $52,00(46,00-62,00)$ & 0,003 \\
\hline Estado Geral de Saúde & $67,00(52,50-82,00)$ & $77,00(68,50-82,00)$ & 0,116 \\
\hline Vitalidade & $55,00(40,00-70,00)$ & $75,00(52,50-90,00)$ & 0,109 \\
\hline Aspectos Sociais & $62,50(50,00-75,00)$ & $75,00(75,00-100,00)$ & 0,019 \\
\hline Aspectos Emocionais & $33,30(0,00-100,00)$ & $66,70(33,30-83,35)$ & 0,230 \\
\hline Saúde Mental & $60,00(42,00-82,00)$ & $84,00(46,00-96,00)$ & 0,091 \\
\hline
\end{tabular}

OBS: $\mathrm{P} \leq 0,05$; Teste Wilcoxon.

Legenda: MPI= mediana pré-intervenção e MPOI= mediana pós-intervenção.

A relação pré e pós-intervenção gerou uma mediana de aumento no escore do SF-36 de 20 pontos $(0,00-65,00)$ na capacidade funcional, 14 pontos $(0,00-79,00)$ em dor, 25 pontos $(0,00-50,00)$ em aspectos sociais.

Valores máximos do escore $(100,00$ pontos) pós-intervenção foram identificados em cinco indivíduos $(38,46 \%)$ no domínio aspectos sociais. Além disso, melhor distribuição interquartil foi identificada no domínio dor pós-intervenção (TABELA 1).

\section{Discussão}

A liberação miofascial neste estudo demonstrou impacto na qualidade de vida, com significância estatística nos domínios capacidade funcional, dor e aspectos sociais. 
Este estudo concorda com um realizado com pacientes que possuem fibromialgia, que também é uma patologia crônica e com limitações funcionais. O estudo citado revela melhora em capacidade funcional, aspectos físicos e aspectos sociais após protocolo de LM. ${ }^{14}$ Efeitos no humor e aumento do limiar nociceptivo também são relatados na literatura secundários a LM.14,23 Outros autores que utilizaram diferentes instrumentos também revelam impacto na qualidade de vida após intervenção miofascial.13,14,23

Contudo, discorrer sobre QV não é uma tarefa simples, já que abrange aspectos emocionais, espirituais e físicos, sendo influenciados por experiências pessoais e coletivas, dependendo ainda de temporalidade e localidade.12 No entanto, muitas pesquisas com o tema têm sido realizadas, valorizando cada vez mais esse indicador biopsicossocial.11-13, 24

$\mathrm{Na}$ literatura são relatados muitos aspectos que podem estar relacionados a um impacto negativo na QV em indivíduos com OA (osteoartrose), como menor capacidade funcional, baixo nível social, pobre comportamento de enfrentamento, impacto negativo econômicofinanceiro, deformidades físicas, dor e menor atividade física gerando, consequentemente, grande impacto na QV. ${ }^{5-8,11,21}$

Indivíduos com IMC elevado, como os da amostra encontrada no estudo, são referenciados por outros autores como predisponentes a uma menor qualidade de vida.8 Desta forma, os indivíduos que não obtiveram resultados mais elevados no escore podem estar relacionados com estes aspectos.

Apesar de no presente estudo não haver significância estatística no domínio aspectos físicos, sabe-se que padrões de aprisionamento fascial estão relacionados a patologias em que partes do corpo deixam de receber estímulos adequados, gerando assim circulação deficiente e limitação no fornecimento de nutrientes para a substância fundamental do tecido conjuntivo. Este déficit em nutrição determina um adensamento fascial, esse que pode tornar os segmentos corporais hipomóveis. A hipomobilidade tende consequentemente a gerar limitações físicas e na capacidade funcional do indivíduo.3,14

Propõe-se na literatura relação estreita entre função física, capacidade funcional e qualidade de vida.810,11 Autores verificam que melhor função física desenvolve melhor qualidade de vida, sendo verificadas melhora em aspectos funcionais, saúde mental, vitalidade e saúde geral do questionário SF-36.24

0 acréscimo em aspectos sociais, encontrado no presente estudo, pode ter sido resultante da melhora da capacidade funcional e da própria comunicação entre os voluntários da pesquisa.30 Foi percebido que passaram a interagir mais entre eles a fim de buscar outros meios para gerar enfrentamento e melhora de sua condição.

A dor como experiência sensorial desagradável, é multidimensional e pode ser influenciada por aspectos físicos ou comportamentais. ${ }^{25}$ Tratamentos que proporcionem melhora destes aspectos geralmente promovem a melhora da dor. Na literatura é proposto que, após terapias manuais, há um alívio sintomático da dor através do relaxamento físico e mental, além de aumento do limiar de dor através da liberação de endorfinas. ${ }^{26} \mathrm{~A}$ LM também possui interação com aumento remoção de substâncias químicas consequentes da lesão, através da ativação circulatória local, reduzindo assim a ativação nociceptora.2

Porém, o fornecimento de informações sobre a sua patologia e dúvidas em relação ao manejo da dor, oferecidas durante as sessões, podem ter mediado ações que reproduzam dor durante o período de intervenção. Este conjunto de informações torna-se um possível viés do estudo, já que a educação em dor tem sido apontada por estudos como ação que reduz dor em pacientes crônicos. ${ }^{25,30}$

Na literatura, muitos benefícios são relatados consequentes da LM, como melhora de dor e função física13,24, restauração postural ${ }^{14}$, estado psicológico de relaxamento ${ }^{23}$, relaxamento muscular por neurorreflexia1 e impacto na qualidade de vida.13-15,23 Achados morfológicos indicam que a LM também possui interação na apoptose celular, atuação 
fibroblástica e na secreção de citocinas 927 , sendo um importante indicador de eficácia das terapias manuais. Estes mesmos autores propõem que a direção, magnitude, duração e frequência da tensão da LM têm importância na definição da homeostase tecidual.

Apesar de tantos benefícios apresentados, ainda não há na literatura um consenso em relação ao seu protocolo, geralmente por divergência de protocolos (tempo de aplicação da técnica, modos de se realizar e freqüências diferentes semanais) ou por relato limitado de seus protocolos em artigos publicados.

Quanto ao tempo de realização, diverge de segundos à dois minutos1, três minutos 28, 10 minutos ${ }^{29}, 20$ minutos $^{29}$ e 90 minutos. ${ }^{14}$ Pode ser realizada focal, nos pontos-gatilho, ou na direção dos feixes musculares, como foi realizada a LM no presente estudo.5,19,20 Pode ainda ser realizada utilizando óleo e associada à aromoterapia, também obtendo melhora em seus marcadores, apesar de não haver significância estatística na maioria dos estudos. ${ }^{29}$

As diferentes metodologias de LM e de instrumentos de avaliação (SF-12, WOMAC ou não referenciada no estudo) ${ }^{13-15,28}$ dificultam a análise mais profunda dos efeitos específicos em cada população, promovendo um viés analítico.

Um viés possível para estes resultados pode ter sido a interferência de outros tratamentos que os pacientes realizaram no mesmo período da aplicação da LM. Os pacientes que possuem patologias crônicas normalmente realizam muitas terapias na tentativa de gerar melhor qualidade de vida.

Os tratamentos realizados na clínica incluíram alongamentos, fortalecimentos e treinos proprioceptivos. Os fortalecimentos e treinos proprioceptivos estimulam estabilidade articular, tendo importância na redução de sintomatologias da OA. No entanto, a massagem terapêutica tem sido apontada em metanálise atual (sobre eficácia de terapias manuais) como indicada com evidência favorável para tratamento de Gonartrose, na impossibilidade de acesso à terapêutica mais efetiva. Sua evidência ainda é inconclusiva por falhas metodológicas e limitado número de artigos disponíveis.5,16

0 alongamento é a técnica que mais se assemelha com a LM, já que possui objetivo de melhorar flexibilidade, reduzindo tensões articulares, sendo indicada como uma das terapêuticas manuais efetivas para artrose de joelho. No entanto, sua ação não é específica fascial, objetivando atuação nas fibras musculares. Autores propõem que a fáscia tem ação mantenedora dos feixes musculares, dando forma e função a esses. Desta forma, para que o tecido muscular seja alongado adequadamente necessita de mobilidade e alongamento fascial complementarmente. ${ }^{1,2}$

Recomenda-se maior controle de variáveis nos próximos estudos, como obter grupo controle, evitar intervenções simultâneas e realizar reavaliações diárias (relatando repercussões a curto e longo prazo), com o objetivo de aumentar a especificidade do estudo.

Com o envelhecimento populacional, aumenta-se progressivamente a incidência de patologias crônicas. Diante disso, aumenta a necessidade de identificação de técnicas que possam ser efetivas para proporcionar melhor qualidade de vida a estes indivíduos. Este estudo busca incentivar a literatura a estudar mais sobre a LM, já que os estudos dispostos na literatura ainda são limitados. Dentre seus benefícios já relatados, a LM é uma técnica que não necessita de muitos acessórios, sendo de baixo custo, com poucas contra-indicações e acessível ao público. Os estudos tem crescido em quantidade recentemente, mostrando que há um interesse global pelo tema.2

\section{Conclusão}

Houve impacto positivo na QV dos indivíduos com gonartrose da amostra, gerando significância estatística em capacidade funcional, dor e aspectos sociais. Apesar de haver muitos estudos que discorram sobre LM, grande parte desses possuem pouca discriminação 
metodológica e/ou qualidade inferior analítica, dificultando a reprodutividade de resultados. Espera-se que este estudo estimule a produção de outros para que se possa obter mais um tratamento coadjuvante para indíviduos com gonartrose, promovendo assim impacto no indicador psicossocial atualmente mais comentado pelos artigos: a qualidade de vida.

\section{Referências}

1- LEITE, J A M; MA'TUTIINO, R.R.B; ARAGÃO, J D. "Efeito da liberação miofascial dos isquiotibiais na amplitude do movimento do quadril", Revista Terapia Manual, 6(25): 154-58. 2008.

2- DIXON, M W. Massagem Miofascial. Serie Physio/fisioterapia prática. Rio de Janeiro-RJ: Guanabara Koogan, 2007.

3- ROLF, I P. Rolfing: A integração das estruturas humanas. São Paulo: 1999.

4- JONES, T A. "Rolfing", Physical Medicine and Rehabilitation Clinics of North America Journal, 15(4): 799-809. 2004.

5- PÉCORA, J R; HERNANDEZ, A J; CAMANHO, G L. Artrose do Joelho gênese e soluções. São Paulo: Atheneu, 2010.

6- WOLLF, A D; PLEGER, B. "Burden of major musculoskeletal conditions. Special Theme of WHO (World Health Organization), Bone and Joint Decade 2000-2010", Bulletin of the World Health Organization, 81(9): 646-56. 2003.

7- RANNOU. F et al. Should aggregate scores of the Medical Outcomes Study 36-Item Short Form Health Survey be used to assess quality of life in knee and hip osteoarthritis? A national survey in primary care. Osteoarthritis and Cartilage. v. 15, n. 9, p. 1013-1018, 2007.

8- ROSEMANN, T. et al. "Association between obesity, quality of life, physical activity and health service utilization in primary care patients with osteoarthritis", International Journal of Behavioral Nutrition and Physical Activity, 8(1): 68. 2008.

9- EAGAN, T S; MELTZER, K R; STANDLEY, P R. "Importance of Strain Direction in Regulating Human Fibroblast Proliferation and Cytokine Secretion: a useful in Vitro Model for soft tissue injury and Manual Medicine Treatments", Journal of Manipulative and Physiological Therapeutics, 30(8): 58492. 2007.

10- DIJK, G M. et al. "Comorbidity, limitations in activities and pain in patients with osteoarthritis of the hip or knee", BMC Musculoskeletal Disorders, 9: p. 95. 2008.

11- ROPMAN, W M. "Associations between chronic disease, age and physical and mental health status", Chronic Diseases in Canada, 29(2): 108-16. 2009.

12- MINAYO, M C S; HARTZ. Z M A; BUSS, P M. "Qualidade de vida e saúde: um debate necessário", Ciência e Saúde Coletiva, 5(1): 7-18. 2000.

13- BALL, T M. "Structural integration-based fascial release efficacy in systemic lupus erythematosus (SLE): two case studies", Journal of Bodywork \& Movement Therapies, 15(2): 217-25. 2011.

14- SÁNCHEZ, A M C. "Benefits of Massage-Myofascial Release Therapy on Pain, Anxiety, Quality of Sleep, Depression, and Quality of Life in Patients with Fibromyalgia", Evidence-Based Complementary and Alternative Medicine, 2011: 1-9. 2011.

15- PERLMAN, A I. et al. "Massage Therapy for Osteoarthritis of the Knee: a randomized controlled trial", Archives of Internal Medicine, 166(22): 2533-38. 2006.

16- BRONFORT, G. et al. "Effectiveness of Manual Therapies: the UK Evidence Report", Chiropratic \& Osteopathy, 18(3): 1-33, 2010.

17- Faculdade de Medicina da Universidade de São Paulo (USP). Laboratório de Epidemiologia e Estatística (LEE). São Paulo. Disponível em: <lee.dante.br/pesquisa/amostragem/calculo_ amostra.html> (Acesso em 18/02/11).

18- World Health Organization. Global BMI Classification. Database on Body Mass Index. (Disponível em: < apps.who.int/bmi/index.jsp?introPage=intro_3.html>, Acesso em 10/10/10). 
19- CLAY, J H; POUNDS, D M. Massoterapia Clínica - integrando anatomia e tratamento. São Paulo: Manole, 2008.

20- BIENFAIT, M. Fáscias e pompages: estudo e tratamento do esqueleto fibroso/ Marcel Bienfait [Tradução Ângela Santos] - São Paulo: Summus, 1999.

21- COOK, C; PIETROBON, R; HEGEDUS, E. “Osteoarthritis and the Impact on Quality of Life Health Indicators", Rheumatology Internacional, 27(4): 315-21. 2007.

23- MORALES, M A. et al. "Psychophysiological Effects of Massage-Myofascial Release After Exercise: A Randomized Sham-Control Study", The Journal of Alternative and Complementary Medicine, 14(10): 1223-29. 2008.

24- AGLAMISA, B; TORANMNB, N F; YAMANC, H. "Change of Quality of Life due to Exercise Training in Knee Osteoarthritis: SF-36 and Woman", Journal of Back and Musculoskeletal Rehabilitation, 22(1): 43-48. 2009.

25-International Association for the Study of Pain edited by Fordyce, W.E. Back Pain in the Workplace: Management of Disability in Nonspecific Conditions. IASP Press. 1995.

26-FURLAN, A.D et al. "Massage for Low-Back pain. Cochrane Database of Systematic Reviews", (Disponível em: <mgsportsmassagetherapy.co.uk/media/Review...pain.pdf $>$, Acesso em 20/02/11).

27-MELTZER, K R. "In Vitro Modeling of Repetitive Motion Injury and Myofascial Release", Journal of Bodywork and Movement Therapies, 14(2): 162-71. 2010.

28-KAIN, J. et al. "Comparison of an indirect tri-planar myofascial release (MFR) technique and a hot pack for increasing range of motion", Journal of Bodywork and Movement Therapies, 15(1): 63-67. 2010.

29- WILKINSON, S; BARNES, K. \& STOREY, L. "Massage for symptom relief in patients with cancer: systematic review", Journal of Advanced Nursing, 63(5): 430-39. 2008.

30- CARVALHO, M G; NOORDHOEK, J; SILVA, M C. "Grupo de orientação a indivíduos acometidos por doenças reumáticas: espaço educativo e terapêutico", Revista Brasileira de Reumatologia, 46(2): 134-36. 2006. 